\title{
P53/MDM2 Co-Expression in Laryngeal Squamous Cell Carcinoma Based on Digital Image Analysis
}

\author{
ARISTEIDIS CHRYSOVERGIS ${ }^{1}$, VASILEIOS PAPANIKOLAOU ${ }^{1}$, EVANGELOS TSIAMBAS ${ }^{2}$, \\ CHARA STAVRAKA ${ }^{3}$, VASILEIOS RAGOS ${ }^{4}$, DIMITRIOS PESCHOS ${ }^{5}$, \\ AMANDA PSYRRI $^{6}$, NICHOLAS MASTRONIKOLIS ${ }^{7}$ and EFTHYMIOS KYRODIMOS ${ }^{1}$ \\ ${ }^{1}$ First Department of Otorhinolaryngology, Head and Neck Surgery, \\ Hippocration Hospital, University of Athens, Athens, Greece; \\ ${ }^{2}$ Department of Immunohistochemistry and Molecular Biology, 401 General Army Hospital, Athens, Greece; \\ ${ }^{3}$ Department of Medical Oncology, Guy's and St Thomas NHS Foundation Trust, London, U.K.; \\ ${ }^{4}$ Department of Maxillofacial, Medical School, University of Ioannina, Ioannina, Greece; \\ ${ }^{5}$ Department of Physiology, Medical School, University of Ioannina, Ioannina, Greece; \\ ${ }^{6}$ Section of Medical Oncology, Department of Internal Medicine, Faculty of Medicine, \\ National and Kapodistrian University of Athens, Attikon University Hospital, Athens, Greece; \\ ${ }^{7}$ Department of Otorhinolaryngology, Medical School, University of Patras, Patras, Greece
}

\begin{abstract}
Background: P53 is a key regulator of genomic stability and function, acting as a tumor suppressor protein. Our aim was to correlate P53 expression with murine double minute 2 (MDM2), a proto-oncogene that interacts with P53 and forms an auto-regulatory pathway, in laryngeal squamous cell carcinoma (LSCC). Materials and Methods: A total of 50 LSCC cases were included in the study. Immunohistochemistry was applied by using antibodies to P53 and MDM2 in the corresponding tissue sections. Protein expression levels for both molecules were measured by implementing a digital image analysis assay (immunostaining intensity levels, densitometric evaluation). Results: Overexpression of P53 protein was observed in 16/50 (32\%) LSCC cases, while 22/50 (44\%) cases strongly expressed MDM2 protein. Interestingly, in 13/50 (26\%) cases, combined overexpression of P53/MDM2 was detected. Overall P53 was strongly positively correlated with MDM2 expression $(p=0.001)$. Both P53 and MDM2 overexpression were significantly correlated with advanced stage of LSCC $(p=0.032$ and $p=0.001$, respectively). Additionally, MDM2 was found to be associated with poorer survival of patients ( $p=0.046)$. Conclusion: Aberrant co-expression of P53 and
\end{abstract}

Correspondence to: Evangelos Tsiambas, Cytopathologist, MD, MSc, PhD, Lecturer Elect in Molecular Cytology, Medical School, University of Athens, 17 Patriarchou Grigoriou E' Street, Ag. Paraskevi, 15341 Athens, Greece. Mobile: +30 6946939414, e-mail: tsiambasecyto@yahoo.gr

Key Words: Larynx, carcinoma, P53, MDM2, immunohistochemistry, digital image analysis.
MDM2 is associated with advanced stage in LSCC. Furthermore, MDM2 overexpression is a frequent and critical genetic event in LSCC and seems to negatively affect survival.

Head and neck squamous cell carcinomas (HNSCC) represent a family of pathological entities demonstrating increased rates worldwide, due to chronic alcohol and tobacco consumption (1). Additionally, persistent infection with oncogenic highrisk human papillomavirus types has been established as another significant etiological factor for HNSCC development and progression (2). Among HNSCCs, laryngeal squamous cell carcinoma (LSCC) is prominent due to its frequency in this anatomical region. Extensive molecular analyses have shown that carcinogenesis in HNSCC is a multistep process of genomic deregulation based on modified cancer stem cells (3). Normal epithelia accumulate gross chromosomal or specific gene instabilities and these are responsible for their neoplastic and finally malignant transformation (4). Overactivation of oncogenes combined with down-regulation of tumor-suppressor genes transforms a normal cell phenotype to a malignant one. Referring to genes that critically modify the cell micro-environment, P53 is of high significance. P53 is a key regulator of genomic stability and function. The gene is located on the short (p) arm of chromosome 17 at position 13.1 (17p13.1). It encodes a nuclear phosphoprotein with a molecular mass of $53 \mathrm{kDa}$ which acts as a transcription factor negatively regulating cell proliferation. It is also involved in a significant number of cell-signaling pathways including the cell cycle, programmed cell death, and DNA repair (5). Normally, wild-type P53 is expressed at a low level in cells, whereas P53 overexpression due to point-mutations is 
frequently detected by immunohistochemistry (IHC) in a broad spectrum of malignancies with different histogenetic origin, including LSCC (6-8). Additionally, murine double minute 2 (MDM2), a proto-oncogene (12q14.3) encoding a nuclear-localized E3 ubiquitin ligase, acts as a major negative regulator in the P53-MDM2 auto-regulatory pathway. MDM2 directly binds to P53, represses its transcriptional activity and promotes its proteasomal degradation (9). Gene amplification is the major mechanism of MDM2 deregulation and its overexpression in breast carcinoma, for example, is correlated with aggressive phenotype. For this reason, novel anti-MDM2 agents have been developed and experimentally applied (10). In the current study, we analyzed P53 and MDM2 at the protein level in LSCC cases in order to determine their coexpression and clinicopathological impact. To our knowledge, this is the first study of P53/MDM2 protein co-expression in HNSCCs, focused especially on LSCC based on a digitized image analysis assay.

\section{Materials and Methods}

Study group. For the purposes of our retrospective analytical study, $50(n=50)$ archival, formalin-fixed and paraffin-embedded tissue specimens (surgical resections or biopsies) of histologically confirmed primary LSCC were used. The specimens were from 45 males and five female patients, all smokers without a positive DNA test or a clear history of human papillomavirus infection. According to their clinical status, patients were treated by chemotherapy (cisplatin-based), radiotherapy, or both combined. The median survival was 43 months. The hospital Ethics Committee consented to the use of these tissues at the Department of Pathology, Hippocration Hospital, University of Athens, Athens, Greece for research purposes, according to the World Medical Association Declaration of Helsinki guidelines (2008 revised 2014). Informed consent was obtained for experimentation with human subjects.

The tissue samples were fixed in $10 \%$ neutral-buffered formalin. Hematoxylin and eosin-stained slides of the corresponding samples were reviewed for confirmation of histopathological diagnoses. All lesions were classified according to the histological typing criteria of World Health Organization (WHO) Tumour Classification (11). Demographic and clinicopathological data of the examined cases are demonstrated in Table I.

Antibodies and IHC. Ready-to-use anti-P53 (clone DO7, dilution 1:40; Dako, Glostrup, Denmark) and anti-MDM2 (clone IF2, dilution 1:40; Novocastra, Newcastle upon Tyne, UK) mouse monoclonal antibodies were applied in the corresponding cases. IHC for the marker expression was carried out on $4-\mu \mathrm{m}$ serial sections of the corresponding tissue blocks. The corresponding slides were deparaffinized and rehydrated. All of them were enzyme digested for $10 \mathrm{~min}$ at $37^{\circ} \mathrm{C}$. The EnVision $^{\mathrm{TM}}+$ system (Dako) detection system was used for the following detection steps. Blocking solution was applied to the slides for $10 \mathrm{~min}$, followed by incubation for $1 \mathrm{~h}$ using the antibodies at room temperature. Following incubation with the secondary antibody for 10 min, diaminobenzidine-tetrahydrocloride $(0.03 \%)$ containing $0.1 \%$ hydrogen peroxide was applied as a chromogen and incubated for 5 min. Sections were counterstained, dehydrated and cover-slipped. The
Table I. Demographic and clinicopathological data of the examined Laryngeal Squamous Cell Carcinoma cases $(n=50)$.

$\mathrm{n}(\%)$

\begin{tabular}{lc}
\hline Gender & \\
Male (median age: 57.5 years) & $45(90 \%)$ \\
Female (median age: 61.5 years) & $5(10 \%)$ \\
Grade & $4(8 \%)$ \\
1 & $19(38 \%)$ \\
2 & $27(54 \%)$ \\
3 & \\
Stage & $4(8 \%)$ \\
II & $25(50 \%)$ \\
III & $21(42 \%)$ \\
IV & \\
Anatomic location & $44(88 \%)$ \\
Supraglottic & $6(12 \%)$ \\
Transglottic & \\
Smoking status & $50(100 \%)$ \\
Yes & $0(0 \%)$ \\
No & \\
Alcohol consumption & $43(86 \%)$ \\
Yes & $7(14 \%)$ \\
No & \\
Therapeutic strategy & $17(34 \%)$ \\
C & $33(66 \%)$ \\
C-RT & \\
Survival (months) & $22(44 \%)$ \\
$0-16$ & $7(14 \%)$ \\
$>16-30$ & $21(42 \%)$ \\
$>30$ & \\
\hline
\end{tabular}

IHC protocol was performed using an automated staining system (I 6000; Biogenex, San Ramon, CA, USA). Breast carcinoma epithelia overexpressing these markers were used as a positive control group. For negative control slides, the primary antibodies were omitted. Predominantly nuclear and slight peri-nuclear cytoplasmic staining was accepted for the markers' positive expression pattern, according to manufacturers' data sheets (Figure 1A and B).

Digital image analysis assay (DIA). P53 and MDM2 protein expression levels were evaluated quantitatively by calculating the corresponding staining intensity levels (densitometric evaluation). DIA was performed using a semi-automated system (hardware: Microscope CX-31, Olympus, Melville, NY, USA; Digital camera, Sony, Tokyo, Japan; Windows XP/NIS-Elements Software AR v3.0, Nikon Corp, Tokyo, Japan). Areas of interest per tissue section were identified (five optical fields at $\times 100$ magnification) and filed in a digital database as snapshots. Measurements were performed by implementing a specific macro (focal nuclear and peri-nuclear cytoplasmic protein expression). Based on an algorithm, staining of normal tissue sections (control) were measured independently and compared to the corresponding values in malignant tissue sections. A broad spectrum of continuous greyscale values (0-255) in the RedGreenBlue (RGB) analysis was available for discriminating different protein expression levels (Figure $1 \mathrm{C}$ and D). Immunostaining intensity values decreasing to 0 represent progressive overexpression of the marker, whereas values increasing to 255 show progressive loss of its staining intensity. 


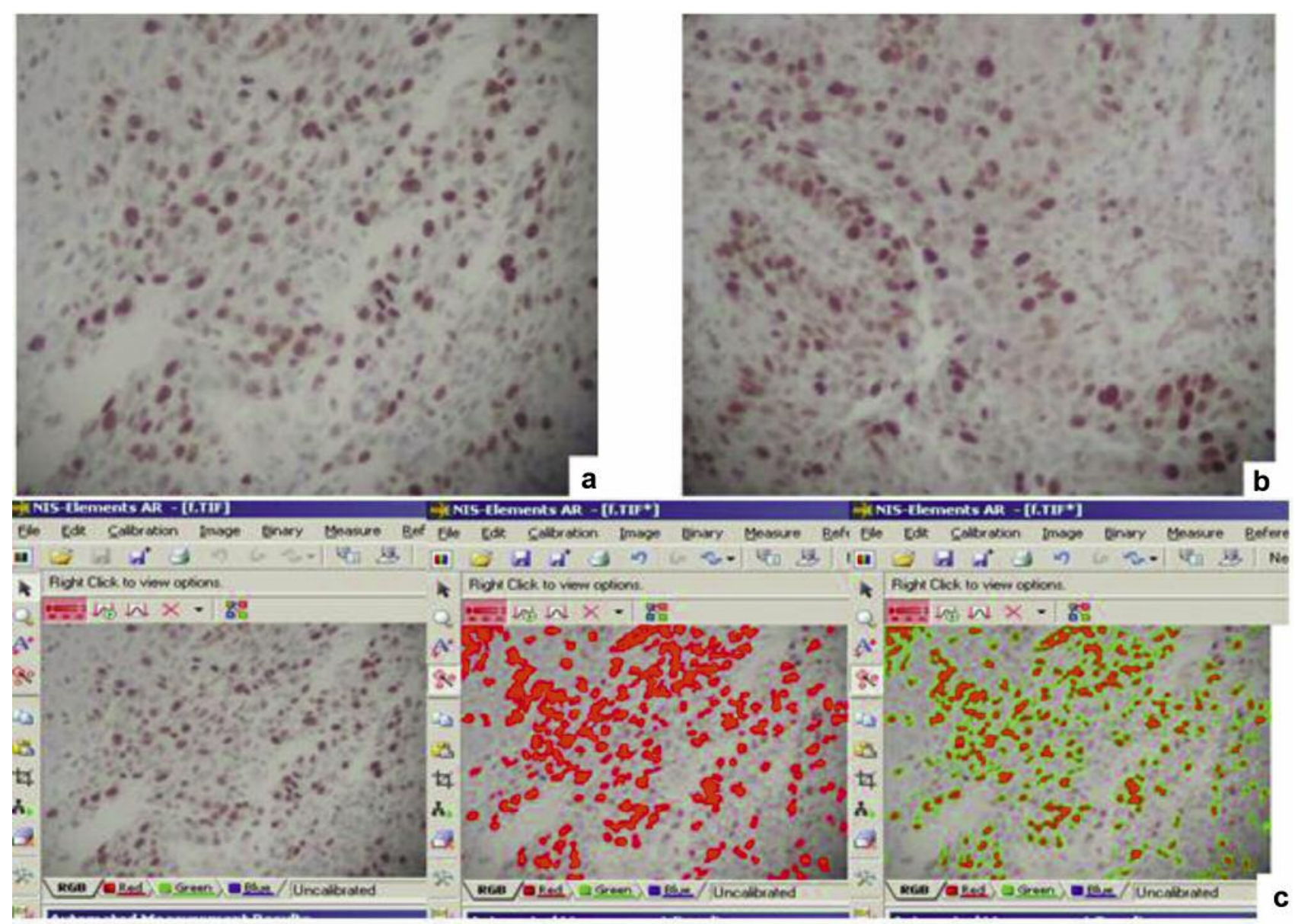

Figure 1. Overexpression of the two markers and digital image analysis in laryngeal squamous cell carcinoma (LSCC): A: P53 and B: murine double minute 2 (MDM2). Note mainly nuclear and cytoplasmic brown staining pattern (diaminobenzidine stain, original magnification: 100X) C: Digital image analysis assay. Red spots represent different expression values of P53-stained nuclei in a case of LSCC. Green loops surrounding red spots represent the final stage of digital analysis providing numerical data (staining intensity values).

Statistical analysis. Descriptive statistics were performed using software package SPSS v25 (IBM, Armonk, NY, USA). Associations between IHC and clinicopathological variables including P53 and MDM2 protein expression levels, gender, tumor grade and stage, anatomical location, and alcohol consumption were extracted by applying Pearson chi-square test. Non-parametric Mann-Whitney $U$ test was used to assess differences in median survival. Two-tailed $p$ values $\leq 0.05$ were considered statistically significant.

\section{Results}

According to the DIA of protein IHC, overexpression of P53 was observed in 16/50 (32\%) LSCC cases, while 22/50 (44\%) cases strongly expressed MDM2 protein. Interestingly, in 13/50 (26\%) cases a co-overexpression of P53 and MDM2 was detected. Additionally, overall P53 was strongly positively correlated with MDM2 expression $(p=0.001)$. Overexpression of P53 and MDM2 were significantly correlated with advanced stage of LSCC ( $p=0.032, p=0.001$, respectively). Additionally, MDM2 expression was found to be negatively associated with survival duration $(p=0.046)$. No other statistical significance was detected for correlation of P53 and MDM2 with gender ( $p=0.712$ and $p=0.259$, respectively), anatomical location ( $p=0.524$ and $p=0.253$, respectively), tumor grade ( $p=0.115$ and $p=0.528$, respectively), therapeutic regimen ( $p=0.194$ and $p=0.622$, respectively) or alcohol consumption ( $p=0.136$ and $p=0.115$, respectively). Results and DIA values are described in Table II.

\section{Discussion}

The P53 mutational landscape demonstrates insertions, deletions, or simpler base substitutions depending on the histological type of malignancy $(12,13)$. As well as P53 mutations leading to abnormal protein expression, wild-type 
Table II. Expression status of P53 and murine double minute 2 (MDM2) in laryngeal squamous cell carcinoma (LSCC) analyzed cases.

\begin{tabular}{|c|c|c|c|c|c|c|c|}
\hline \multirow[t]{2}{*}{ Clinicopathological parameter } & \multirow[b]{2}{*}{$\mathrm{n}$} & \multicolumn{2}{|c|}{ P53 } & \multirow[t]{2}{*}{$p$-Value } & \multicolumn{2}{|c|}{ MDM2 } & \multirow[t]{2}{*}{$p$-Value } \\
\hline & & $\mathrm{OE}$ & LE & & $\mathrm{OE}$ & LE & \\
\hline LSCC & 50 & $16 / 50(32 \%)$ & $34 / 50(68 \%)$ & & $22 / 50(44 \%)$ & $28 / 50(56 \%)$ & \\
\hline \multicolumn{8}{|l|}{ Gender } \\
\hline Male & 45 & $14 / 50(28 \%)$ & $31 / 50(62 \%)$ & 0.712 & $21 / 50(42 \%)$ & $24 / 50(48 \%)$ & 0.259 \\
\hline Female & 5 & $2 / 50(4 \%)$ & $3 / 50(6 \%)$ & & $1 / 50(2 \%)$ & $4 / 50(8 \%)$ & \\
\hline \multicolumn{8}{|l|}{ Grade } \\
\hline 1 & 4 & $0 / 50(0 \%)$ & $4 / 50(8 \%)$ & 0.115 & $1 / 50(2 \%)$ & $3 / 50(6 \%)$ & 0.528 \\
\hline 2 & 19 & $4 / 50(8 \%)$ & $15 / 50(30 \%)$ & & $10 / 50(20 \%)$ & $9 / 50(18 \%)$ & \\
\hline 3 & 27 & $12 / 50(24 \%)$ & $15 / 50(30 \%)$ & & $11 / 50(22 \%)$ & $16 / 50(32 \%)$ & \\
\hline \multicolumn{8}{|l|}{ Stage } \\
\hline II & 4 & $1 / 50(2 \%)$ & $3 / 50(6 \%)$ & 0.032 & $2 / 50(8 \%)$ & $12 / 50(24 \%)$ & 0.001 \\
\hline III & 25 & $4 / 50(8 \%)$ & $21 / 50(42 \%)$ & & $2 / 50(4 \%)$ & $13 / 50(26 \%)$ & \\
\hline IV & 21 & $11 / 50(8 \%)$ & $10 / 50(20 \%)$ & & $18 / 50(36 \%)$ & $3 / 50(6 \%)$ & \\
\hline \multicolumn{8}{|l|}{ Survival (months) } \\
\hline $0-16$ & 22 & $10 / 50(20 \%)$ & $12 / 50(24 \%)$ & 0.154 & $13 / 50(26 \%)$ & $9 / 50(18 \%)$ & 0.046 \\
\hline$>16-30$ & 7 & $1 / 50(2 \%)$ & $6 / 50(12 \%)$ & & $2 / 50(4 \%)$ & $5 / 50(10 \%)$ & \\
\hline$>30$ & 21 & $5 / 50(10 \%)$ & $16 / 50(32 \%)$ & & $7 / 50(14 \%)$ & $14 / 50(28 \%)$ & \\
\hline
\end{tabular}

P53 OE: P53 overexpression, staining intensity values $\leq 131$ (spectrum between 79 and 131), LE: P53 low expression values between 142 and 169 ; MDM2 OE: MDM2 overexpression staining intensity values $\leq 128$ (spectrum between 71 and 128), LE: MDM2 low expression values >139 (spectrum between 139 and 172).

P53 is influenced by two main inhibitors: MDM2 and MDM4. The role of MDM4 is critical because it inhibits the transcriptional activity of $P 53$, enhancing also the ability of MDM2 to target P53 for degradation (14). Overexpression of these molecules is mediated mainly by gene amplification in a broad spectrum of solid malignancies, including LSCC (15-17). Another epigenetic mechanism of deregulation of the P53/MDM2 auto-regulatory pathway is the aberrant alternative splicing of mRNA precursors. This process leads to abnormal protein expression that promotes cell growth, local invasion, and metastasis by enhancing oncogenes and down-regulates suppressor genes (18). Additionally, the role of post-transcriptional regulators, termed microRNAs, seems to be significant for regulating the P53/MDM2 dependent pathway. In particular, positive feedback loops involving micro-RNAs such as $m i R-192, m i R-34 a$, and $m i R-29 a$ have been already identified in genetic signatures of LSCC (19).

In the current study, we analyzed P53/MDM2 complex at the protein expression level in LSCC tissues. A significant subset of the examined tumors demonstrated cooverexpression, whereas in the rest, elevated MDM2 expression inversely correlated with P53 level. Interestingly, overexpression of the two molecules was significantly correlated with an aggressive phenotype (advanced stage) in the examined cases. Furthermore, MDM2 overactivation was found to be associated with reduced survival of patients. Similarly, other studies have shown that overexpression of mutated P53 is frequently detected in a significant proportion of LSCC tissues $(20,21)$. Another study group investigated the potential role of P53 and B-cell lymphoma 2 (BCL2) (gene locus: 18q21.33), an anti-apoptotic factor suppressing and blocking apoptotic signal transduction, as potential reliable molecular markers for patients with LSCC (22). Based on assays of IHC, polymerase chain reaction, DNA ploidy and Sphase fractions, they concluded that aberrant P53 expression due to mutation should be used as a marker for predicting treatment response rates in patients with LSCC. Concerning natural agents that might be promoters of P53-mediated apoptotic activity in LSCC, a study group proposed Boschniakia rossica polysaccharide (BRP). Treating Hep2 laryngeal carcinoma cells with BPR at different concentrations they observed that a high apoptotic rate was established due to P53 and enhancement of caspase complex (especially caspase3) (23). Additionally, another molecular study showed that induction of P53 activity based on miR-30b overexpression enhanced adenovirus-mediated P53 cancer gene therapy for patients with LSCC (24). Regarding MDM2 gene up-regulation in LSCC, interesting molecular data have been derived by exploring the role of its specific polymorphisms. One study group showed that MDM2 rs769412 and rs937283 polymorphisms might be used as genetic markers for increased laryngeal carcinoma risk, especially in cases characterized by chronic alcohol consumption (25). Similarly, another genetic study focused on the role of MDM2 -309 T/G single nucleotide polymorphism in a specific population (Chinese Han) analyzing samples by applying pyrosequencing technique. They concluded that the majority of patients with LSCC MDM2 -309TG genotype demonstrated better phenotype (low 
stage and metastatic potential) compared to those with TT, characterized also by a reduced plasma MDM2 level, which is considered as a favorable prognostic factor (26). Because MDM2 overactivation negatively modifies the biological behavior of this malignancy, and the response rate to chemoradiotherapy regimens, it should potentially be considered as a marker of LSCC aggressiveness $(27,28)$. For this reason, small-molecule inhibitors of MDM2, such as nutlin-3, are promising agents for enhancing radiosensitivity of LSCC cells by inducing P53 apoptotic activity (29). Finally, in the current IHC analysis, we applied a DIA protocol for measuring the P53 and MDM2 protein expression levels. This approach provides a totally objective estimation of IHC-based densitometric values in contrast to subjective conventional IHC screening under bright-field microscopy. Especially combined with novel grids in cytological, mainly immunostained, slides, the current technique is a reliable tool for diagnostic and research approaches in the field of accurate, improved evaluation of IHC slides as we have already reported $(30,31)$.

\section{Conclusion}

Overexpression of $M D M 2$ oncogene, mainly due to gene amplification, is a frequent and critical genetic event in LSCC. In contrast, expression of mutated P53 protein combined with MDM2 leads to an aggressive phenotype (advanced stage). Novel targeted therapeutics should be developed with the aim of blocking MDM2 activity combined with up-regulation of P53.

\section{Disclosure}

The Authors declare no conflict of interest.

\section{Authors' Contributions}

Aristeidis Chrysovergis: Clinical advisor, researcher. Vasileios S. Papanikolaou: Clinical advisor, researcher. Evangelos Tsiambas: Researcher, article writing. Chara Stavraka: Clinical advisor, statistical analysis. Vasileios Ragos: Academic advisor. Dimitrios Peschos: Academic advisor. Amanda Psyrri: Academic advisor. Efthymios Kyrodimos: Academic advisor, article writing. Nicholas Mastronikolis: Case stratification, statistical analysis.

\section{References}

1 Grégoire V, Lefebvre J-L, Licitra L and Felip E: Squamous cell carcinoma of the head and neck: EHNS-ESMO-ESTRO clinical practice guidelines for diagnosis, treatment and follow-up. Ann Oncol 21: 184-186, 2010. PMID:20555077. DOI: 10.1093/ annonc/mdq 185

2 Termine N, Panzarella V, Falaschini S, Russo A, Matranga D, Lo Muzio L, and Campisi G: HPV in oral squamous cell carcinoma $v s$. head and neck squamous cell carcinoma biopsies: A meta-analysis (1988-2007). Ann Oncol 19(10): 1681-1690, 2008. PMID: 18558666 . DOI: $10.1093 /$ annonc/mdn372
3 Graziano A, d'Aquino R, Tirino V, Desiderio V, Rossi A and Pirozzi G: The stem cell hypothesis in head and neck cancer. J Cell Biochem 103(2): 408-412, 2008. PMID: 17546610. DOI: $10.1002 / \mathrm{jcb} .21436$

4 Lea IA, Jackson MA, Li X, Bailey S, Peddada SD and Dunnick JK: Genetic pathways and mutation profiles of human cancers: Site- and exposure-specific patterns. Carcinogenesis 28(9): 18511058, 2007. PMID: 17693665. DOI:10.1093/carcin/bgm176

5 Purvis JE, Karhohs KW, Mock C, Batchelor E, Loewer A and Lahav G: P53 dynamics control cell fate. Science 336(6087): 1440-1444, 2012. PMID: 22700930. DOI: 10.1126/science. 1218351

6 Dumay A, Feugeas JP, Wittmer E, Lehmann-Che J, Bertheau P, Espie M, Plassa LF, Cottu P, Marty M, André F, Sotiriou C, Pusztai L and de Thé $\mathrm{H}$ : Distinct tumor protein P53 mutants in breast cancer subgroups. Int J Cancer 132: 1227-1231, 2013. PMID: 22886769. DOI: 10.1002/ijc. 27767

7 Silwal-Pandit L, Vollan HK, Chin SF, Rueda OM, McKinney S, Osako T, Quigley DA, Kristensen VN, Aparicio S, BørresenDale AL, Caldas C and Langerød A: TP53 mutation spectrum in breast cancer is subtype specific and has distinct prognostic relevance. Clin Cancer Res 20(13): 3569-3580, 2014. PMID: 24803582. DOI: 10.1158/1078-0432.CCR-13-2943

8 Osman I, Sherman E, Singh B, Venkatraman E, Zelefsky M, Bosl G, Scher H, Shah J, Shaha A, Kraus D, Cordon-Cardo C and Pfister DG: Alteration of P53 pathway in squamous cell carcinoma of the head and neck: Impact on treatment outcome in patients treated with larynx preservation intent. J Clin Oncol 20(13): 2980-2987, 2002. PMID: 12089228. DOI:10.1200/JCO. 2002.06 .161

9 Qin JJ, Wang W, Voruganti S, Wang H, Zhang WD and Zhang R: Inhibiting NFAT1 for breast cancer therapy: New insights into the mechanism of action of MDM2 inhibitor JapA. Oncotarget 6(32): 33106-33119, 2015. PMID: 26461225. DOI:10.18632/ oncotarget.5851

10 Wang W, Qin JJ, Voruganti S, Voruganti S, Srivenugopal KS, Nag S and Patil S: The pyrido[b]indole MDM2 inhibitor SP-141 exerts potent therapeutic effects in breast cancer models. Nat Commun 5: 5086-5090, 2014. PMID: 25271708. DOI: 10.1038/ ncomms6086

11 El-Naggar AK, Chan JKC, Grandis JR, Takata T and Slootweg PJ: World Health Organization Classification of Head and Neck Tumours. Fourth Edition. Lyon: IARC Press; 2017.

12 Santarius T, Shipley J, Brewer D, Stratton MR and Cooper CS: A census of amplified and overexpressed human cancer genes. Nat Rev Cancer 10: 59-64, 2010. PMID: 20029424. DOI: $10.1038 / \mathrm{nrc} 2771$

13 Walerych D, Napoli M, Collavin L and Del Sal G: The rebel angel: Mutant P53 as the driving oncogene in breast cancer. Carcinogenesis 33: 2007-2017, 2012. PMID: 22822097. DOI: $10.1093 /$ carcin/bgs 232

14 Lam S, Lodder K, Teunisse AF, Rabelink MJ, Schutte M and Jochemsen AG: Role of MDM4 in drug sensitivity of breast cancer cells. Oncogene 29: 2415-2426, 2010. PMID: 20140020. DOI: $10.1038 /$ onc.2009.522

15 Kriegmair MC, Balk M, Wirtz R, Steidler A, Weis CA, Breyer J, Hartmann A, Bolenz C and Erben P: Expression of the P53 inhibitors MDM2 and MDM4 as outcome predictor in muscleinvasive bladder cancer. Anticancer Res 36(10): 5205-5213, 2016. PMID: 27798881. DOI:10.21873/anticanres.11091 
16 Wade M, Li YC and Wahl GM: MDM2 MDMX and P53 in oncogenesis and cancer therapy. Nat Rev Cancer 13: 83-96, 2013. PMID: 23303139. DOI: $10.1038 / \mathrm{nrc} 3430$

17 Freier CP, Stiasny A, Kuhn C, Mayr D, Alexiou C, Janko C, Wiest I, Jeschke U and Kost B: Immunohistochemical evaluation of the role of P53 mutation in cervical cancer: Ser-20 P53mutant correlates with better prognosis. Anticancer Res 36(6): 3131-3137, 2016. PMID: 27272838.

18 Inoue K and Fry EA: Aberrant splicing of the DMP1-ARFMDM2-P53 pathway in cancer. Int J Cancer 139(1): 33-41, 2016. PMID: 26802432. DOI: 10.1002/ijc.30003

19 Moore R, Ooi HK, Kang T, Bleris L and Ma L: miR-192mediated positive feedback loop controls the robustness of stress-induced P53 oscillations in breast cancer cells. PLoS Comput Biol 11(12): e1004653, 2015. PMID: 26642352. DOI: 10.1371/journal.pcbi.1004653

20 Malm IJ, Rooper LM, Bishop JA, Ozgursoy SK, Hillel AT, Akst LM and Best SR: Molecular and immunologic analysis of laryngeal squamous cell carcinoma in smokers and non-smokers. Am J Otolaryngol 40(2): 213-217, 2019. PMID: 30553600. DOI: 10.1016/j.amjoto.2018.11.009

21 Owusu-Afriyie O, Owiredu WKBA, Owusu-Danquah K, LarsenReindorf R, Donkor P, Acheampong E, Quayson SE, Prince MEP, McHugh JB, Donkor P, Merajver SD and Brenner: Expression of immunohistochemical markers in nonoropharyngeal head and neck squamous cell carcinoma in Ghana. PLoS One 13(8): e0202790, 2018. PMID: 30138436. DOI: 10.1371/journal.pone.0202790

22 Sahoo R, Chittibabu V, Patil G, Rao S, Thakur S, Dhondalay G, Kulkarni AJ, Banerjee A, Ajaikumar BS, Korlimarla A, Nargund A, Niti RN, Gopinath KS, Prabhudesai S and Raghavendra RM: Relationship between molecular markers and treatment response in a retrospective cohort of Indian patients with primary carcinoma of the larynx. Oral Oncol 45(12): e216-221, 2009. PMID: 19729336. DOI: 10.1016/j.oraloncology.2009.07.013

23 Yao C, Cao X, Fu Z, Tian J, Dong W, Xu J, An K, Zhai L and Yu J: Boschniakia rossica polysaccharide triggers laryngeal carcinoma cell apoptosis by regulating expression of BCL-2, caspase-3, and P53. Med Sci Monit 23: 2059-2064, 2017. PMID: 28455995. DOI: $10.12659 / \mathrm{msm} .901381$

$24 \mathrm{Li} \mathrm{L}$ and Wang B: Overexpression of microRNA-30b improves adenovirus-mediated P53 cancer gene therapy for laryngeal carcinoma. Int J Mol Sci 15(11): 19729-19740, 2014. PMID: 25356506. DOI:10.3390/ijms 151119729

24 Wang H and Ma K: Association between MDM2 rs769412 and rs937283 polymorphisms with alcohol drinking and laryngeal carcinoma risk. Int J Clin Exp Pathol 8(6): 7436-7440, 2015. PMID: 26261649.
25 Zhou J, Liu F, Zhang D, Chen B, Li Q, Zhou L Lu LM and Tao L: Significance of MDM2-309 polymorphisms and induced corresponding plasma MDM2 levels in susceptibility to laryngeal squamous cell carcinoma. DNA Cell Biol 33(2): 8894, 2014. PMID: 24325471. DOI: 10.1089/dna.2013.2220

26 Hassumi-Fukasawa MK, Miranda-Camargo FA, Guimarães MC, Simões RT, Donadi EA, Soares CP and Soares EG: Possible implication of MDM2 as a prognostic marker in invasive laryngeal carcinoma. Eur Arch Otorhinolaryngol 269(7): 1795-1804, 2012. PMID: 22310835. DOI: 10.1007/s00405-012-1937-3

27 Chrysovergis A, Papanikolaou VS, Tsiambas E, Ragos V, Peschos D and Kyrodimos E: Digital analysis of BCL2 expression in laryngeal squamous cell carcinoma. Anticancer Res 39(3): 12531257, 2019. PMID: 30842155. DOI: 10.21873/anticanres.13235

28 Ganly I, Talbot S, Carlson D, Viale A, Maghami E, Osman I, Sherman E, Pfister D, Chuai S, Shaha AR, Kraus D, Shah JP, Socci ND and Singh B: Identification of angiogenesis/metastases genes predicting chemoradiotherapy response in patients with laryngopharyngeaI carcinoma. J Clin Oncol 25(11): 1369-1376, 2007. PMID: 17416856.

29 Arya AK, El-Fert A, Devling T, Eccles RM, Aslam MA, Rubbi CP, Vlatković N, Fenwick J, Lloyd BH, Sibson DR, Jones TM and Boyd MT: Nutlin-3, the small-molecule inhibitor of MDM2, promotes senescence and radiosensitises laryngeal carcinoma cells harbouring wild-type P53. Br J Cancer 103(2): 186-195, 2010. PMID: 20588277. DOI: 10.1038/sj.bjc.6605739

30 Faratzis G,Tsiambas E, Rapidis AD, Machaira A, Xiromeritis K and Patsouris E: VEGF and Ki-67 expression in squamous cell carcinoma of the tongue: An immunohistochemical and computerized image analysis study. Oral Oncol 45(7): 584-548, 2009. PMID: 18804402. DOI: 10.1016/j.oraloncology. 2008.08.002

31 Tsiambas E, Riziotis C, Mastronikolis NS, Peschos D, Mortakis A, Kyroysis G, Mastronikolis SN, Batistatou A, Lazaris AC, Patsouris E and Ragos V: Comparative p16IKN4Aexpression in laryngeal carcinoma and cervical cancer precursors: A real-time grid-based immunocytochemistry analysis. Anticancer Res 38(10): 5805-5810, 2018. PMID: 30275203. DOI: 10.21873/ anticanres. 12920
Received May 21, 2019

Revised June 19, 2019

Accepted June 20, 2019 